\title{
Bioactive anthraquinones found in plant foods interact with human serum albumin and inhibit the formation of advanced glycation endproducts
}

\author{
Weixi Liu ${ }^{\mathrm{a}, \mathrm{b}, \mathrm{c}}$, Ang Cai ${ }^{\mathrm{b}, \mathrm{c}}$, Rachel Carley ${ }^{\mathrm{b}}$, Robert Rocchio ${ }^{\mathrm{b}}$, Zoe M. Petrovas ${ }^{\mathrm{b}}$, Cassie A. Chartier $^{\mathrm{b}}$, \\ Xiaofeng Meng ${ }^{\mathrm{d}}$, Jianyu Su${ }^{\mathrm{d}}$, Bongsup P. Cho ${ }^{\mathrm{b}}$, Joel A. Dain ${ }^{\mathrm{c}}$, \\ Hang $\mathrm{Ma}^{\mathrm{a}, \mathrm{b}^{*}}$ and Navindra P. Seeram ${ }^{\mathrm{b}^{*}}$
}

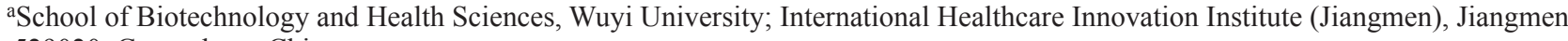
529020, Guangdong, China

bepartment of Biomedical and Pharmaceutical Sciences, College of Pharmacy, University of Rhode Island, Kingston, RI 02881, USA

${ }^{\mathrm{c}}$ Department of Chemistry, University of Rhode Island, Kingston, RI 02881, USA

dSchool of Food Science and Engineering, South China University of Technology; Guangdong Province Key Laboratory for Green Processing of Natural Products and Product Safety; Overseas Expertise Introduction Center for Discipline Innovation of Food Nutrition and Human Health (111 Center), Guangzhou 510640, China

*Corresponding author: Hang Ma, School of Biotechnology and Health Sciences, Wuyi University; International Healthcare Innovation Institute (Jiangmen), Jiangmen 529020, Guangdong, China. Tel: 401-874-7654; E-mail: hang_ma@uri.edu; Navindra P. Seeram, Department of Biomedical and Pharmaceutical Sciences, College of Pharmacy, University of Rhode Island, Kingston, RI 02881, USA. Tel: 401874-9367; E-mail: nseeram@uri.edu
\end{abstract}

DOI: $10.31665 /$ JFB. 2018.4169

Received: November 14, 2018; Revised received \& accepted: December 20, 2018

Abbreviations: AGEs, advanced glycation endproducts; AG, aminoguanidine; CD, circular dichroism; GO, Glyoxal; HSA, human serum albumin; ITC, isothermal titration calorimetry; MGO, methylglyoxal; RCS, reactive carbonyl species.

Citation: Liu, W., Cai, A., Carley, R., Rocchio, R., Petrovas, Z.M., Chartier, C.A., Meng, X., Su, J., Cho, B.P., Dain, J.A., Ma, H., and Seeram, N.P. (2018). Bioactive anthraquinones found in plant foods interact with human serum albumin and inhibit the formation of advanced glycation endproducts. J. Food Bioact. 4: 130-138.

\begin{abstract}
The glycation of human serum albumin (HSA) plays a critical role in the development of many disorders. Herein, the anti-glycation effects of several natural dietary anthraquinone derivatives including aloin, aloe-emodin, chrysophanol, emodin, physcion, and rhein were evaluated. The anthraquinones at $100 \mu \mathrm{M}$ reduced fructose-, methylglyoxal-, and glyoxal-induced HSA glycation by $21 \%$ (aloe-emodin) to $92 \%$ (aloin), $17 \%$ (physcion) to $94 \%$ (emodin), and 16\% (anthraquinone) to 67\% (emodin), respectively. These anthraquinone derivatives also protected HSA by maintaining its free amino acid residues and secondary protein structure as characterized by circular dichroism. The mechanisms of their anti-glycation effects were investigated using rhein as a representative anthraquinone. The interactions between rhein and HSA were evaluated by biophysical characterizations, namely, isothermal titration calorimetry and UV-vis spectroscopy as well as computational modeling. Our findings suggest that the anti-glycation effects of these anthraquinones may be attributed to their binding capacity and stabilization of the HSA protein structure.
\end{abstract}

Keywords: Anthraquinone; Rhein; Advanced glycation endproducts (AGEs); Human serum albumin (HSA); Circular dichroism (CD); Isothermal titration calorimetry (ITC). 


\section{Introduction}

Human serum albumin (HSA) is the most abundant protein in circulation and possesses a wide variety of physiological functions. It is a carrier protein which transports biomolecules including hormones, ions, fatty acids, and several drugs (Fanali et al., 2012). It is also the predominant antioxidant in plasma due to its free radical and metal ion trapping abilities (Anraku et al., 2013; Roche et al., 2008). However, being constantly exposed to numerous chemicals in circulation, HSA is highly susceptible to glycation, which is the non-enzymatic reaction between proteins and sugars. Glycated albumin accounts for $80 \%$ of the glycated proteins in circulation and this proportion may increase up to $90 \%$ in severely diabetic patients (Anguizola et al., 2013; Raghav and Ahmad, 2014). The physiological impact of glycated albumin is enormous. First, glycation is among one of the major mechanisms attributed to the structural and functional alteration of HSA. As a result, the antioxidant property and binding capacity of HSA can be significantly reduced or impaired when it is glycated. Second, the glycation of HSA can lead to the formation of advanced glycation end-products (AGEs), which play a major role in numerous disorders including diabetic complications. Therefore, AGEs inhibitors are being investigated as potential treatments for AGEs-induced diabetic complications and considerable research efforts have been dedicated to identifying AGEs inhibitors from dietary natural products and medicinal plants (Peng et al., 2011; Wu et al., 2011).

Anthraquinone (9,10-dioxoanthracene) derivatives are commonly found in many medicinal plants and food stuffs. For instance, aloe (Aloe vera) is a valuable ingredient for the food, pharmaceutical, and cosmetic industries (Eshun and He, 2004) and it contains high levels of anthraquinones including aloe-emodin, rhein, chrysophanol, and other anthraquinone derivatives (Choi and Chung, 2003). Garden rhubarb (Rhubarb rhabarbarum) is a vegetable containing anthraquinones including rhein, emodin, aloeemodin, physcion, and chrysophanol (He et al., 2009; Koyama et al, 2007). Similarly, Senna obtusifolia (Chinese senna, or American sicklepod) is a medicinal plant containing anthraquinones including aloe-emodin, aloin, rhein, and emodin (Dave and Ledwani, 2012; Sob et al., 2010). Its seed, known as cassiae semen, is commonly consumed as a roasted tea in Korea and China. The water extract of cassiae semen has been developed as functional food such as a slimming tea which contains six anthraquinones including aurantioobtusin, rhein, aloe-emodin, emodin, chrysophanol, and physcion (Wang et al., 2016). As a chemical class, anthraquinones have been studied for their anti-diabetic activities including antihyperglycemic effects (Arvindekar et al., 2015) and inhibitory effects against carbohydrate hydrolyzing enzymes, $\alpha$-glucosidase and amylase (Adisakwattana et al., 2010; Arvindekar et al., 2015). However, while anthraquinones have been reported to show inhibitory effects against the formation of AGEs (Xie and Chen, 2013; Yoo et al., 2010), these mechanism(s) of inhibition still remain unclear.

Our research group has had a long interest in the identification of AGEs inhibitors from dietary natural products, including medicinal plants and functional foods, and investigating their mechanisms of action (Liu et al., 2014, 2016, 2017; Ma et al., 2015, 2015, 2016, 2018; Sun et al., 2016). Interestingly, our studies have shown that while several of these dietary phytochemicals inhibit the formation of AGEs, they exert these effects via varying mechanisms including reactive carbonyl species trapping effects (Liu et al., 2014), free radical scavenging capacity (Ma et al., 2016), and protective effects on the protein structure (Ma et al., 2015). In this contribution, we investigated the inhibitory effects of several dietary anthraquinone derivatives including rhein, aloin, emodin, chrysophanol, physcion, and aloe-emodin against the glycation of HSA. The mechanisms of the inhibitory effects of these anthraquinones were further investigated, using rhein as a representative anthraquinone, in biophysical assays (isothermal titration calorimetry and UV-vis spectroscopy) and computational modeling experiments.

\section{Materials and methods}

\subsection{Materials}

Anthraquinone, rhein, D-fructose, aminoguanidine hydrochloride (AG), glyoxal (GO), methylglyoxal (MGO), 2,2-diphenyl-1-picrylhydrazyl, 2,3-dimethylquinoxaline (DQ), 1,2-phenylenediamine (PD), butylated hydroxytoluene (BHT), trifluoroacetic acid (TFA), HPLC-grade methanol, and fluorescamine were purchased from Sigma-Aldrich Chemical Co. (St. Louis, MO, USA). Aloin, emodin, chrysophanol, physcion, and aloe-emodin were purchased from Xi' an Natural-Field Bio-Technique Co. (Xi'an, China). Fatty acid free human serum albumin (HSA) was purchased from EMD Millipore Co. (Billerica, MA, USA). Solid black 96-well plates were purchased from Corning Inc. (Corning, NY, USA).

\subsection{HSA-fructose glycation assay}

The HSA-fructose assay was used to evaluate the inhibitory effects of the anthraquinones on protein glycation following methods described previously (Liu et al., 2014). Briefly, $10 \mathrm{mg} / \mathrm{mL}$ of HSA and $100 \mathrm{mM}$ D-fructose solutions were prepared under sterile conditions and incubated at $37^{\circ} \mathrm{C}$ for 21 days. In the treated samples, the concentration of the anthraquinones was adjusted to $100 \mu \mathrm{M}$. A synthetic anti-glycation agent, aminoguanidine (AG), at an equivalent concentration of $100 \mu \mathrm{M}$, served as the positive control. After 21 days of incubation, the intrinsic fluorescence level of each sample was determined using a Spectra Max M2 spectrometer (Molecular Devices, Sunnyvale, CA, USA). The excitation and emission wavelengths were 360 and $435 \mathrm{~nm}$, respectively. The inhibition level was calculated using the following equation: $\%$ inhibition $=$ [1-(fluorescence intensity of solution with treatment/fluorescence intensity of negative control solution)] $\times 100 \%$.

\subsection{HSA-MGO and HSA-GO glycation assay}

Reactive carbonyl compounds, methylglyoxal (MGO) and glyoxal (GO), were used to induce HSA glycation following previously reported methods (Ma et al., 2018). In brief, each reaction mixture consisted of $10 \mathrm{mg} / \mathrm{mL}$ of HSA and $5 \mathrm{mM}$ of MGO or $1 \mathrm{mM}$ of GO. Treatments included the anthraquinones at a concentration of 100 $\mu \mathrm{M}$ and the positive control, $\mathrm{AG}$, at an equivalent concentration. Following incubation at $37^{\circ} \mathrm{C}$ for $72 \mathrm{~h}$, samples were measured for MGO- and GO-induced glycation level on a Spectra Max M2 spectrometer (Molecular Devices, Sunnyvale, CA, USA) at excitation and emission wavelengths of 360 and $435 \mathrm{~nm}$, respectively. The inhibition level was calculated using the following equation: $\%$ inhibition $=[1$-(fluorescence intensity of solution with treatment/fluorescence intensity of negative control solution) $] \times 100 \%$.

\subsection{Fluorescamine assay}

To determine free amino groups of HSA before and after glycation, 
the fluorescamine assay was performed following previously reported methods with minor modifications (Yaylayan et al., 1992). Briefly, $2 \mathrm{mg} / \mathrm{mL}$ of the HSA-fructose mixture $(5 \mu \mathrm{L})$ obtained in the aforementioned HSA-fructose assay (section 2.2) were mixed with $1 \mathrm{mM}$ fluorescamine reagent $(50 \mu \mathrm{L})$ and $0.1 \mathrm{M}$ phosphate buffer $(145 \mu \mathrm{L})$. The mixtures were incubated in the dark for 10 mins and the intrinsic fluorescence was determined at excitation and emission wavelengths of 390 and $490 \mathrm{~nm}$, respectively.

\subsection{Free radical and reactive carbonyl species trapping assays}

The free radical trapping capacities of the anthraquinones were evaluated using the 2,2-diphenyl-1-picrylhydrazyl (DPPH) assay as previously reported (Ma et al., 2018). Each test sample was dissolved in $10 \%$ dimethyl sulfoxide (DMSO) to yield a stock solution of $1 \mathrm{mM}$ concentration which was then diluted with $50 \%$ aqueous methanol to desired concentrations ranging from $31-1000 \mu \mathrm{M}$. The test samples $(100 \mu \mathrm{L})$ and the DPPH solution $(10 \mathrm{mg} / \mathrm{mL}$ in $50 \%$ aqueous methanol; $100 \mu \mathrm{L}$ ) were mixed in a 96-well plate and incubated for $30 \mathrm{~min}$ in the dark. The absorbance was determined at 517 nm using a SpectraMax M2 (Molecular Devices, Sunnyvale, CA, USA) plate reader. The MGO trapping capacities of the anthraquinones were evaluated by measuring the derivatized MGO residual (2-methylquinoxaline) using a high performance liquid chromatography (HPLC) method as previously reported by our laboratory (Liu et al., 2014). Briefly, each test sample $(100 \mu \mathrm{M} ; 250 \mu \mathrm{L})$ was added to a mixture consisting of MGO $(5 \mathrm{mM} ; 150 \mu \mathrm{L})$, PD (20 $\mathrm{mM} ; 50 \mu \mathrm{L})$, and DQ $(5 \mathrm{mM} ; 50 \mu \mathrm{L})$ and incubated for $30 \mathrm{~min}$ at room temperature. The derivatized 2-methylquinoxaline was quantified by HPLC analyses as previously reported (Liu et al., 2014).

\subsection{Circular dichroism (CD) analysis}

The glycated HSA solutions obtained from the HSA-fructose assay (section 2.2) were subjected to $\mathrm{CD}$ analysis to investigate the protective effects of the anthraquinones on HSA structure. CD measurements were performed on a Jasco J-720 spectropolarimeter (Tokyo, Japan). All samples were diluted with $0.2 \mathrm{M}$ phosphate buffer to adjust the protein concentration to $0.5 \mathrm{mg} / \mathrm{mL}$ prior to the $\mathrm{CD}$ measurements. Each solution $(300 \mu \mathrm{L})$ was then transferred to a quartz cuvette with $1 \mathrm{~mm}$ path length and the CD spectrum was obtained from 190 to $250 \mathrm{~nm}$ with 5 consecutive scans. The interpretation of results was then performed by the Spectra Manager software (Tokyo, Japan).

\subsection{UV-vis absorbance spectroscopy}

UV-vis spectroscopy experiments were conducted to investigate the interactions of HSA and rhein. UV-vis spectra were obtained on a Cary100 Bio UV-Vis spectrophotometer (Santa Clara, CA, USA) equipped with a $6 \times 6$ multi-cell chamber $(1.0 \mathrm{~cm}$ path length) and connected with a built-in Peltier temperature controller. Rhein samples (at concentrations of 25, 50, and $100 \mu \mathrm{M}$ ) were prepared in phosphate-buffered saline (PBS; 0.1 M). HSA samples were prepared in PBS at concentrations of $0.125,0.25$, and $0.5 \mu \mathrm{g} /$ $\mathrm{mL}$. Each sample was scanned forward/reverse for five times.

\subsection{Isothermal titration calorimetry (ITC) analysis}

Isothermal titration calorimetry (ITC) analysis was conducted to investigate the interactions between HSA and rhein. The binding affinity $(\mathrm{Ka})$, the number of binding sites (stoichiometry, N), and the binding enthalpy $(\Delta \mathrm{H})$ were measured by titrating rhein into native HSA. A VP-ITC micro calorimeter from MicroCal (Northampton, MA, USA) was used to record the binding affinity of rhein $(1 \mathrm{mM})$ with native HSA $(0.015 \mathrm{mM})$ in $0.1 \mathrm{mM}$ PBS buffer $(\mathrm{pH}$ 7.0) using previously reported method with modifications (Ma, Wang, et al., 2015). All of the samples were degassed extensively for 20 mins by using ThermoVac (MicroCal, Northampton, MA, USA). Each ITC experiment consisted of 16 injections and each injection released $16 \mu \mathrm{L}$ of rhein solution into the HSA cell over $32 \mathrm{sec}$ with a $240 \mathrm{sec}$ delay between each injection at the $307 \mathrm{rpm}$ stirring speed at $25{ }^{\circ} \mathrm{C}$. The integrated and normalized areas of raw data were plotted as $\mathrm{kcal} / \mathrm{mol}$ of injectant against the molar ratio. The titration data was analyzed by using ORIGIN 7.0 software (Northampton, MA, USA) with a single-site binding model and $\Delta H, K_{\mathrm{a}}$ and $N$ were obtained from the interactions between rhein and HSA.

\subsection{Molecular docking}

The crystallographic structure of HSA was obtained from the Protein Data Bank [RSCB ID: 1AO6]. The computational docking between HSA and rhein was performed using the Autodock Vina function built into UCSF Chimera version 1.13.1. Two separate $30 \times 30 \times 30$ angstrom grid box was created for docking computations. Broyden-Fletcher-Goldfarb-Shanno (BFGS) method was used to measure the total negative force, torque, and torque projections acting on a ligand. The outputs for each grid box with the most negative scores were selected and two outputs were presented in BIOVA Discovery Studio with HSA to view 2D ligandreceptor interactions.

\section{Results and discussion}

\subsection{Anthraquinones inhibit the formation of D-fructose-, MGO-, and GO-induced HSA glycation}

Three well known glycation inducers, D-fructose, methylglyoxal (MGO), and glyoxal (GO), were separately used to initiate the glycation of HSA. Anthraquinone and its natural derivatives including aloe-emodin, aloin, chrysophanol, emodin, physcion, and rhein (chemical structures shown in Figure 1a) were individually added to the reaction mixtures to evaluate their anti-glycation effects on HSA. As shown in Figure 1b, the anthraquinones reduced HSA glycation at a concentration of $100 \mu \mathrm{M}$. Among the seven anthraquinone derivatives, aloin showed the highest inhibitory effect in the HSA-fructose assay which reduced the formation of fluorescent glycation products by $92.3 \%$, followed by rhein $(80 \%)$, emodin $(79 \%)$, anthraquinone $(68 \%)$, chrysophanol $(68 \%)$, physcion $(44 \%)$ and aloe-emodin $(21 \%)$. The synthetic anti-glycating agent, aminoguanidine (AG), used as a positive control at equivalent concentration $(100 \mu \mathrm{M})$, only reduced HSA glycation by $10 \%$. In the HSA-MGO assay (Figure 1c), the most potent anti-glycation effect was observed with emodin $(100 \mu \mathrm{M})$, which reduced HSAMGO glycation by $94.0 \%$, followed by aloin (56\%), rhein $(45 \%)$, aloe emodin $(33 \%)$, chrysophanol $(23 \%)$, physcion $(19 \%)$ and anthraquinone $(17 \%)$. All of the anthraquinones showed stronger anti-glycation activities compared to the positive control, AG, which had an inhibition capacity of $13 \%$ at the equivalent concentration $(100 \mu \mathrm{M})$. Similarly, the anthraquinones (at $100 \mu \mathrm{M}$ ) 
<smiles>O=C1c2cccc(O)c2C(=O)c2c(O)cc(CO)cc21</smiles>

Aloe emodin<smiles>O=C1c2c(O)cccc2C(c2cc(CO)cc(O)c2O)C2C(O)C(O)C(O)C(O)C12</smiles>

Aloin<smiles>Cc1cc(O)c2c(c1)C(=O)c1cccc(O)c1C2=O</smiles>

Anthraquinone
Chrysophanol<smiles>Cc1cc(O)c2c(c1)C(=O)c1cc(O)cc(O)c1C2=O</smiles>

Emodin<smiles>COc1cc(O)c2c(c1)C(=O)c1cc(C)cc(O)c1C2=O</smiles>

Physcion<smiles>O=C(O)c1cc(O)c2c(c1)C(=O)c1cccc(O)c1C2=O</smiles>

Rhein

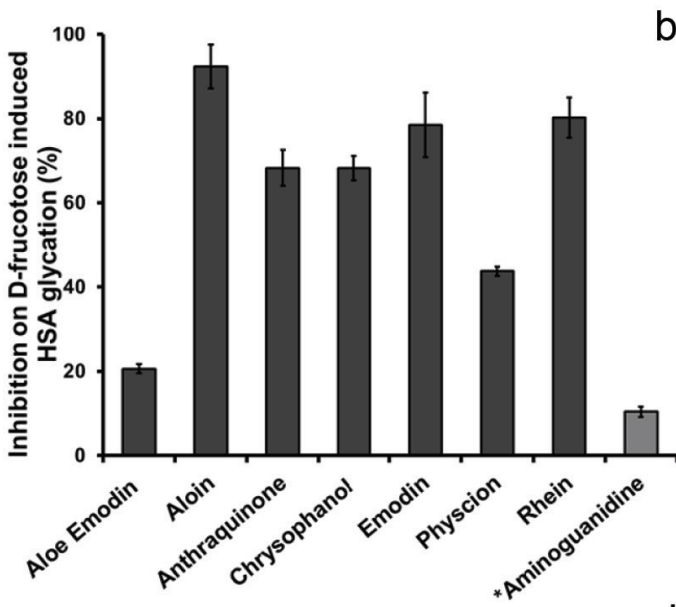

b
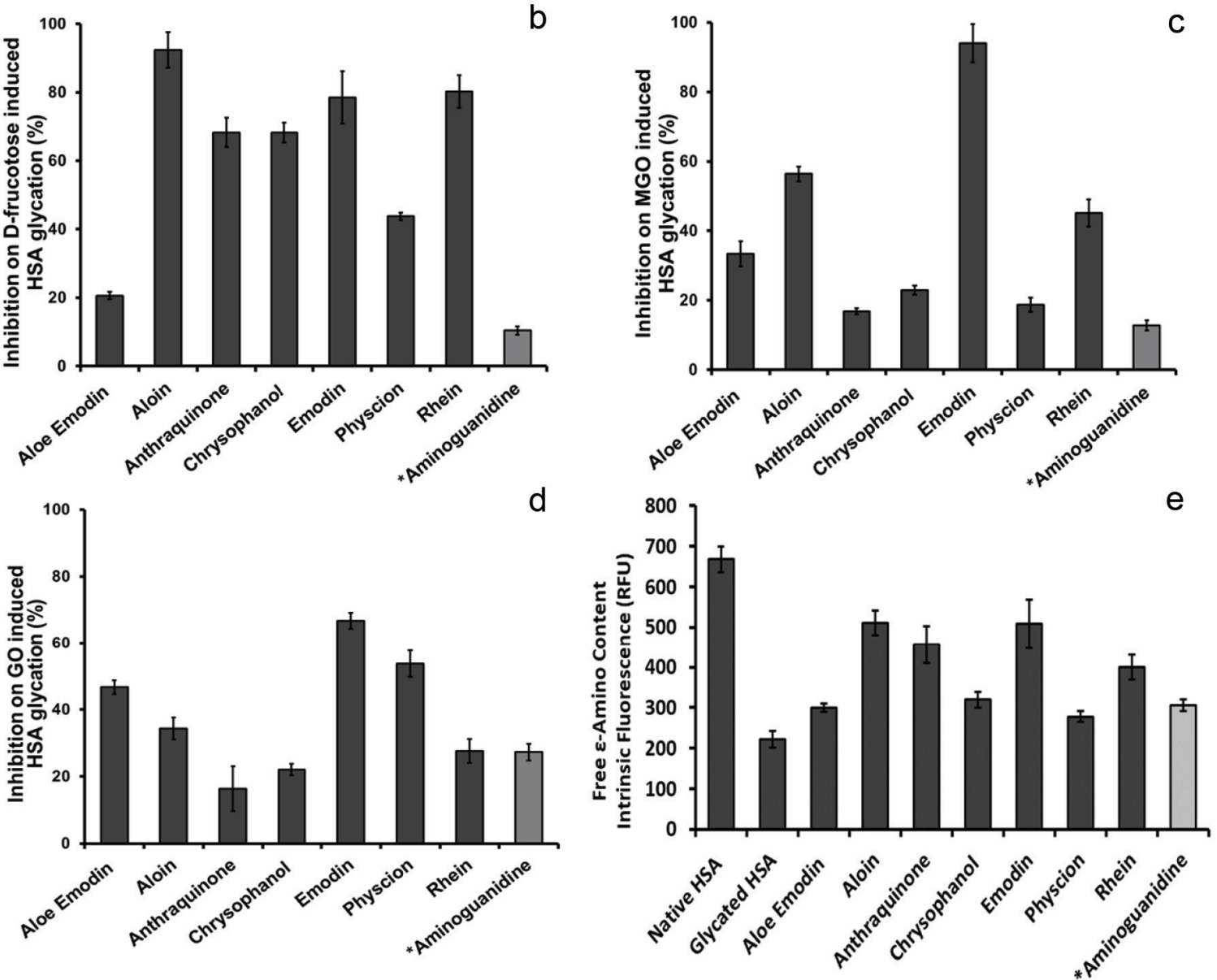

Figure 1. Chemical structures of anthraquinone derivatives (a). The intrinsic fluorescence characterization of effects of the anthraquinones (at $100 \mu \mathrm{M}$ ) on the formation of HSA AGEs with different inducers including fructose (b), methylglyoxal (MGO) (c), and glyoxal (GO) (d). The inhibitory effects of the anthraquinones (at $100 \mu \mathrm{M}$ ) on HSA side chain modification induced by fructose (e). All data points represent the average of triplicate measurements with the bars at each point representing the respective standard derivation. *Aminoguanidine, AG (100 $\mu \mathrm{M})$, served as a positive control.

showed inhibitory effects on HSA glycation in the HSA-GO assay. Emodin showed the highest inhibition capacity of $67 \%$ followed by physcion (54\%), aloe-emodin (47\%), aloin (34\%), rhein (28\%), chrysophanol (22\%), and anthraquinone (16\%). At the same concentration, AG had a moderate activity with an inhibition capacity of $27 \%$. 
Table 1. Scavenging effects of anthraquinones on free radicals in the DPPH assay and reactive carbonyl species in the MGO trapping assay

\begin{tabular}{|c|c|c|}
\hline Sample & Free radical scavenging capacity; $\mathrm{IC}_{50}(\mu \mathrm{M})$ & MGO trapping capacity (\%) \\
\hline aloe emodin & $80.1 \pm 1.7$ & n.d. ${ }^{a}$ \\
\hline emodin & $125.7 \pm 3.3$ & 7.2 \\
\hline aloin & $502.9 \pm 6.9$ & 11.7 \\
\hline chrysophanol & $>1000$ & n.d. \\
\hline rhein & $>1000$ & 2.3 \\
\hline anthraquinone & $>1000$ & n.d. \\
\hline physcion & $>1000$ & n.d. \\
\hline $\mathrm{BHT}$ & $397.5 \pm 4.8$ & - \\
\hline$A G^{c}$ & - & 96.1 \\
\hline
\end{tabular}

${ }^{a}$ n.d. $=$ not detectable at a concentration of $100 \mu \mathrm{M}$. ${ }^{\mathrm{b}} \mathrm{BHT}$ : butylated hydroxytoluene, positive control for the DPPH assay. ${ }^{\mathrm{C}} \mathrm{AG}$ : aminoguanidine, positive control for the MGO trapping assay.

The anti-glcyation effects of the anthraquinone derivatives were further evaluated in fluorescamine assay which measures the levels of non-glycated amino acids residues in HSA. The concentration of the free $\varepsilon$-amino groups in the protein was determined by intrinsic fluorescence. The fructose-glycated HSA showed a pronounced decrease in free $\varepsilon$-amino content when comparing to native non-glycated HSA, which is due to structural modification of amino acid residues when HSA was exposed to glycating agents. As shown in Figure 1e, the free $\varepsilon$-amino content in the glycated HSA was reduced by $66.8 \%$ as compared to native HSA. However, in anthraquinones $(100 \mu \mathrm{M})$ treated groups, the free $\varepsilon$-amino contents of fructose-glycated HSA were maintained as follows: aloin (77\%), emodin (76\%), anthraquinone (68\%), rhein $(60 \%)$, chrysophanol $(48 \%)$, aloe-emodin $(45 \%)$, and physcion $(42 \%)$. The positive control, AG, maintained the free $\varepsilon$-amino content of fructose-glycated HSA at $46 \%$. This observation confirmed that these anthraquinone derivatives were able to attenuate the glycation-induced modification of HSA amino acid residues. Our observations are in agreement with a previously reported study wherein anthraquinones including aloe-emodin, emodin, and chrysophanol from an Aloe vera extract inhibited the formation of glucose-induced AGEs in a bovine serum albumin model (Kang et al., 2017). However, that study was conducted at a reaction temperature of $70{ }^{\circ} \mathrm{C}$ (Kang et al., 2017) whilst our current data was obtained at a physiologically relevant reaction temperature of $37^{\circ} \mathrm{C}$. Although all of the anthraquinone derivatives tested in our study showed promising anti-glycation activities, in general, their inhibition potency varied in the different glycation models with the different glycation inducers namely, fructose, $\mathrm{MGO}$, and GO. This may be attributed to the varying chemical structures of the anthraquinones, such as the numbers of hydroxyl groups on the benzene rings (see Figure 1). For example, an anthraquinone with three hydroxyl groups (namely, emodin) showed the highest inhibitory activities in the MGO- and GO-induced glycation models, whereas anthraquinones with two hydroxyl groups (namely, aloe-emodin, aloin, chrysophanol, physcion, and rhein) or with the absence of a hydroxyl group (anthraquinone) showed weaker anti-glycation activities. However, this preliminary structure and activity relationship observation was not applicable in the glycation models with the less reactive inducer (D-fructose) wherein anthraquinone showed moderate activity and the highest activity was observed with the treatment of aloin. Therefore, our observations suggest that the number/s of hydroxyl group/s in these anthraquinones may be critical to their anti-glycation effects but further studies on structure and activity relationships are warranted to confirm this.

\subsection{Anthraquinones show weak activities in free radical and methylglyoxal (MGO) scavenging assays}

Free radical scavenging activity and reactive carbonyl species (RCS) trapping capacity are well established as important mechanisms for the anti-glycation effects of several AGEs inhibitors (Liu et al., 2014; Ma et al., 2016). Therefore, the mechanisms of the anti-glycation effects of the anthraquinones were first investigated in these aforementioned assays. As shown in Table 1, aloe-emodin and emodin had moderate free radicals scavenging activity with $\mathrm{IC}_{50}$ values of 80.1 and $125.7 \mu \mathrm{M}$, respectively, in the DPPH assay. The free radical scavenging activity of aloin was comparable to the positive control, butylated hydroxytoluene $\left(\mathrm{BHT} ; \mathrm{IC}_{50}=502.9 \mu \mathrm{M}\right.$ vs $\left.\mathrm{IC}_{50}=397.5 \mu \mathrm{M}\right)$. The other anthraquinone derivatives showed weak free radical scavenging effects with $\mathrm{IC}_{50}$ values greater than $1000 \mu \mathrm{M}$. In the RCS trapping assay, aloin, emodin, and rhein (at $100 \mu \mathrm{M}$ ) showed weak MGO trapping capacity (with a trapping rate of $11.7,7.2$, and $2.3 \%$, respectively) while the other anthraquinones did not show any MGO trapping activity. The positive control, AG, showed an MGO trapping activity rate of $96.1 \%$ at the equivalent concentration of $100 \mu \mathrm{M}$. The weak MGO trapping capacities of the anthraquinone derivatives observed herein were in agreement with a previously reported study wherein a Polygonum cuspidatum extract showed promising MGO trapping activity but this was not attributed to its constituent anthraquinones including emodin, physcion, and their glycoside derivatives (Tang et al., 2013). Therefore, the weak free radical scavenging and MGO trapping effects of these anthraquinone derivatives suggested that their inhibition of RCS-induced HSA glycation were via other mechanisms rather than through their direct RCS trapping effects. Next, we conducted further investigations to elucidate the mechanisms of action of the anthraquinone derivatives.

\subsection{Anthraquinones reduce the alterations of the secondary structure of HSA}

It has been reported that several phytochemicals impart anti-glycation effects by maintaining the structure of proteins (Joglekar et al., 2013; Ma et al., 2015). The glycation of globular proteins, such 


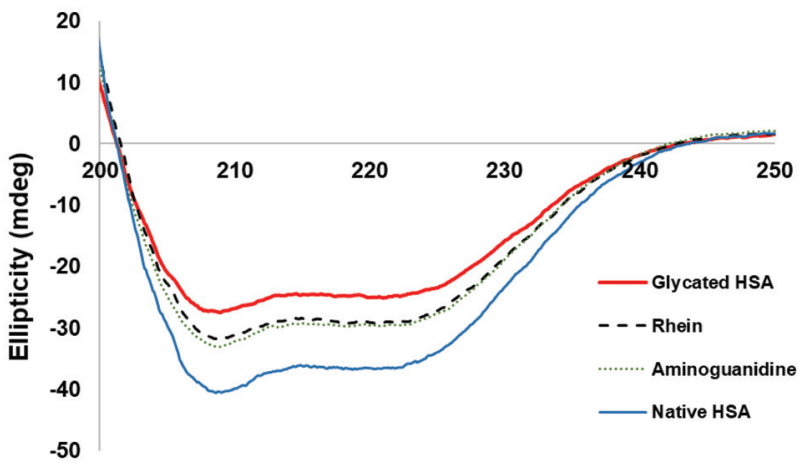

Wavelength $(\mathrm{nm})$

Figure 2. Protective effects of the anthraquinones (at $100 \mu \mathrm{M}$ ) on the secondary structures of HSA against glycation-induced structural changes as characterized by circular dichroism (CD) experiments. Aminoguanidine, AG, (at $100 \mu \mathrm{M})$ served as the positive control.

as HSA, can induce conformational alterations of the protein structures resulting in a decrease in helical structures and an increase in beta-sheets and random coils. Herein, the secondary structures of native HSA and glycated HSA were evaluated by circular dichroism (CD) analyses. The CD spectra obtained from native HSA, glycated HSA, and anthraquinones-treated HSA solutions showed two prominent peaks at around $208 \mathrm{~nm}$ and $222 \mathrm{~nm}$. The signals at these wavelengths showed a positive correlation with the levels of $\alpha$-helical structures. As shown in Figure 2, the CD spectrum of glycated HSA showed the lowest signal response as compared to the spectrum of rhein-treated glycated HSA, indicating that rhein reduced the glycation-induced confirmation alteration of HSA by retaining the $\alpha$-helical structures of HSA (66.1 vs 32.5\%). Similar effects were observed in the CD spectra of other anthraquinonestreated HSA solutions. The anthraquinones protected the secondary structures of HSA by maintaining $\alpha$-helical structures content as follows: emodin (57.7\%), chrysophanol (56.9\%), aloin (46.3\%), anthraquinone $(44.8 \%)$, rhein $(44.3 \%)$, aloe-emodin $(43.5 \%)$, and physcion $(43.0 \%)$, while the positive control, AG, maintained the $\alpha$-helical structures at $46.6 \%$ (Table 2). These data suggest that these anthraquinones protect HSA protein structure and reduce its structural change during glycation. This is similar to our pre-

\section{Time (min)}

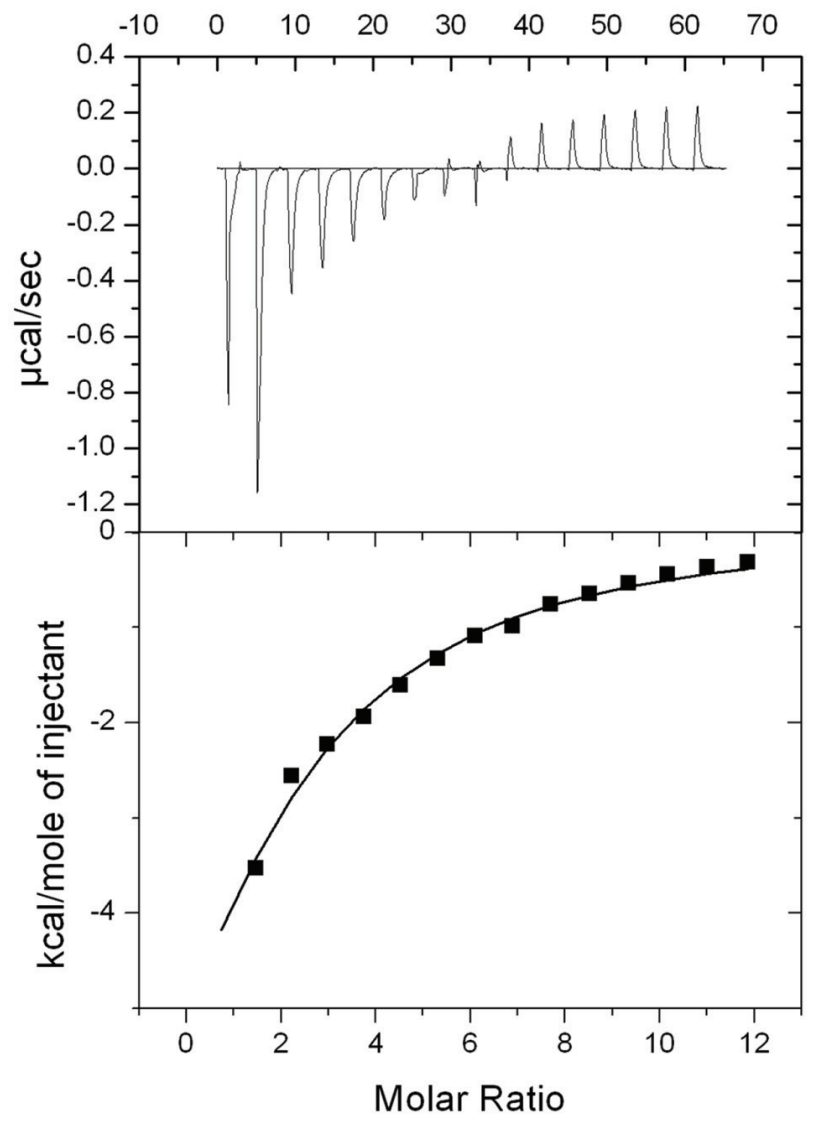

Figure 3. Thermodynamic binding capacity of rhein and HSA obtained by isothermal titration calorimetry (ITC). Raw data plot of heat flow against time for the titration of rhein into HSA.

viously reported study wherein a polyphenol, namely, pentagalloyl glucose, inhibited protein glycation by protecting its secondary structures (Ma et al., 2015). In addition, previously reported spectroscopic and chromatographic studies demonstrated that an-

Table 2. Protective effects of anthraquinones on the secondary structure of HSA protein as the percentages of the HSA secondary structures including $\alpha$-helix, $\beta$-sheet, and irregular

\begin{tabular}{llll}
\hline Secondary structures & $\boldsymbol{\alpha}$-Helix (\%) & $\boldsymbol{\beta}$-Sheet (\%) & Irregular (\%) \\
\hline native HSA & 66.1 & 25.3 & 8.6 \\
glycated HSA & 32.5 & 49.7 & 17.8 \\
aloe emodin & 43.5 & 42.2 & 14.3 \\
aloin & 46.3 & 41.8 & 11.9 \\
anthraquinone & 44.8 & 41.6 & 13.6 \\
chrysophanol & 56.9 & 34.3 & 8.8 \\
emodin & 57.7 & 33 & 9.3 \\
physcion & 43.0 & 41.9 & 15.1 \\
rhein & 44.3 & 39.1 & 16.6 \\
aminoguanidine & 46.6 & 38.7 & 14.7 \\
\hline
\end{tabular}

The raw $C D$ data were obtained from three replicates of experiments and their average values were used to calculate the percentage of the secondary structures. 
Table 3. Thermodynamic parameters for ligand (rhein) binding to HSA

\begin{tabular}{|c|c|c|c|c|c|c|}
\hline Ligand & N [rhein/HSA] & $K a\left[104 \mathrm{M}^{-1}\right]$ & c value & $\Delta G\left[104 \mathrm{kcal} \mathrm{mol}^{-1}\right]$ & $\Delta H\left[104 \mathrm{kcal} \mathrm{mol}^{-1}\right]$ & $-T \Delta S\left[104 \mathrm{kcal} \mathrm{mol}^{-1} \mathrm{~K}^{-1}\right]$ \\
\hline rhein & 1.9 & 2.1 & 0.6 & -0.6 & -1.2 & 0.6 \\
\hline
\end{tabular}

thraquinones were able to interact with bovine serum albumin and stabilize the conformation of that protein (Bi et al., 2005). In order to investigate the interactions and binding mechanism of HSA and anthraquinones, rhein was selected as a representative anthraquinone for further studies including isothermal titration calorimetry (ITC) and UV-vis spectroscopy. We selected rhein because it is among one of the most extensively studied anthraquinones implicated with wide range of biological effects (Agarwal et al., 2000; Yen et al., 2000).

\subsection{Rhein binds to HSA as measured by Isothermal Titration Calorimetry (ITC)}

The thermodynamic binding properties between rhein and HSA were determined by ITC measurements. The top panel of Figure 3 illustrates raw ITC data where each injection of rhein into the HSA solution is reflected by each peak in the binding isotherm. A titration curve of the amount of heat released per injection of rhein as a function of the molar ratio was constructed as shown in the bottom panel of Figure 3. The thermodynamic parameters for rhein binding to HSA are summarized in Table 3. Rhein had an approximate 2 to 1 ligand-protein binding ratio $(\mathrm{N}$ value $=1.9)$ with HSA indicating that two molecules of rhein bind to one molecule of HSA. The negative titration peaks and a negative enthalpy value $\left(\Delta \mathrm{H}=-1.2 \times 10^{4} \mathrm{kcal} / \mathrm{mol}\right)$ revealed that the binding interactions between rhein and HSA were exothermic. Rhein and HSA had a binding constant $(\mathrm{Ka})$ of $2.1 \times 10^{4} \mathrm{M}^{-1}$ and a hyperbolic curve with a low c value (0.6). A negative $\Delta \mathrm{H}$ value $\left(-0.6 \times 10^{4} \mathrm{kcal} /\right.$ $\mathrm{mol})$ and a positive $-\mathrm{T} \Delta \mathrm{S}$ value $\left(0.6 \times 10^{4} \mathrm{kcal} / \mathrm{mol}\right)$ suggested that the interactions between rhein and HSA were predominantly driven by an exothermic process. Although the interactions between rhein and HSA have been previously investigated using fluorescent spectroscopic methods (Bi et al., 2005; Li et al., 2007; Vargas et al., 2004), the thermodynamic parameters of the binding reaction between rhein and HSA remain unclear. Therefore, in the current study, the data from the ITC experiment provided useful insights of the thermodynamic interactions between rhein and HSA and the ligand-protein binding ratio was determined to be $2: 1$. This is similar to the observations from another study using ITC measurement in which a small molecule, namely, diclofenac, a non-steroidal anti-inflammatory drug, was also able to bind to HSA in a 2:1 ratio (Bou-Abdallah et al., 2016).

\subsection{Rhein interacts with HSA as measured by UV-vis spectros- copy}

The interactions between rhein and HSA amino acid residues were further supported by data from UV-vis spectroscopic assays. The UV-vis spectra of HSA (at $0.25 \mathrm{mg} / \mathrm{mL}$ ) with the absence and presence of rhein (at concentrations of 25,50 , and $100 \mu \mathrm{M}$ ) are shown in Figure 4a. The UV-vis spectrum of HSA alone showed a shoulder peak at $278 \mathrm{~nm}$. As the concentrations of rhein added to the HSA solution increased, the absorbance of this peak increased with a slight shift to higher wavelengths $(2 \mathrm{~nm})$. In addition, the rheinHSA complex showed major peaks at $228 \mathrm{~nm}$ and $434 \mathrm{~nm}$ and the absorbance of these peaks also increased as the concentrations of rhein increased. These observations indicate that rhein interacted with the tryptophan residue of HSA and changed the conformation of the HSA protein, which is in agreement with data obtained from the CD experiments and previously reported data (Ding et al., 2012). To further confirm this observation, the spectra of rhein $(25$ $\mu \mathrm{M}$ ) with different concentrations of HSA (at $0.125,0.25$, and 0.5 $\mathrm{mg} / \mathrm{mL}$ ) were obtained (Figure $4 \mathrm{~b}$ ). As expected, the HSA-rhein complex showed a red shift as the concentrations of HSA increased suggesting that rhein interacted with HSA in a concentration-dependent manner.

\subsection{Computational modeling of the rhein-HSA interaction}

The data of interactions between rhein and HSA obtained from biophysical experiments were complemented by computational modeling study. The possible binding sites of rhein on HSA were visualized in Figure 5. The overall structure of HSA as a globular protein is shown in Figure 5a. There are three homologous domains (I, II, and III) in HSA and each one can be divided into two subdomains, A and B, containing six (A) and four (B) $\alpha$-helices, respectively (He and Carter, 1992). It has been well studied that two common binding sites on HSA are hydrophobic pockets of subdomains IIA and IIIA. Based on the rank of the binding energy, rhein was able to bind to the subdomain IIA of HSA (Figure 5b), which is in agreement with previous studies (Li et al., 2007; Vargas et al., 2004). Possible binding force between rhein and HSA, including hydrophobic and electrostatic interaction, are demonstrated in Figure $5 \mathrm{c}$. Strong hydrogen bonds were formed between rhein and arginine 222 and 257, glutamine 153, and tyrosine 150 . In addition, the planar structures of rhein (benzene rings) facilitated the $\pi-\Sigma$ interactions with amino acid residues including leu-
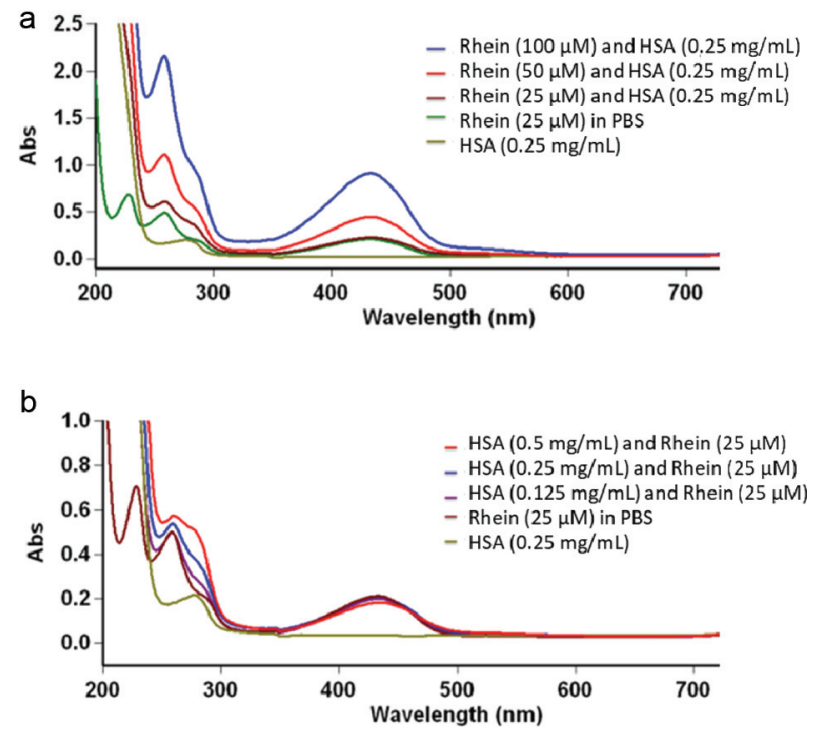

Figure 4. The UV-visible absorption spectra of HSA $(0.25 \mathrm{mg} / \mathrm{mL})$ with rhein at concentrations ranging from 25 to $100 \mu \mathrm{M}(\mathrm{a})$ and rhein $(25 \mu \mathrm{M})$ with HSA at concentrations ranging from 0.125 to $0.5 \mathrm{mg} / \mathrm{mL}$ (b). 
a

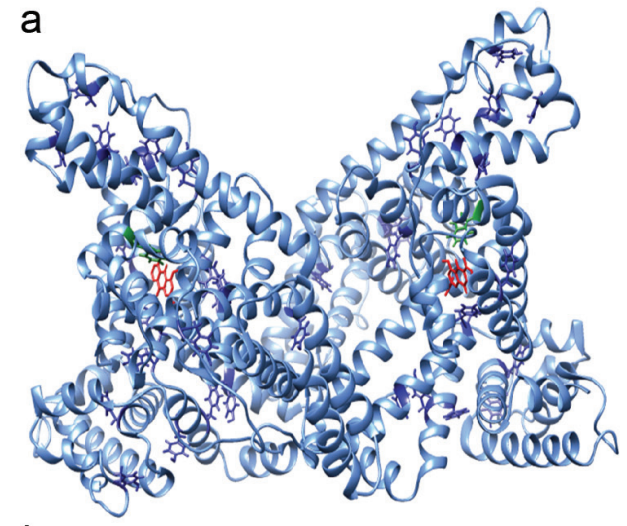

b

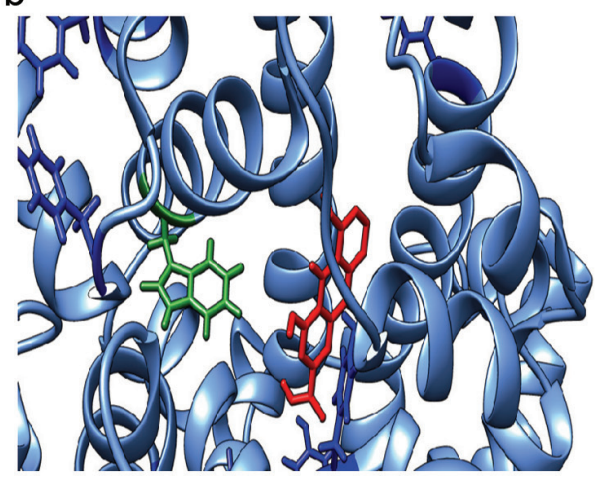

C

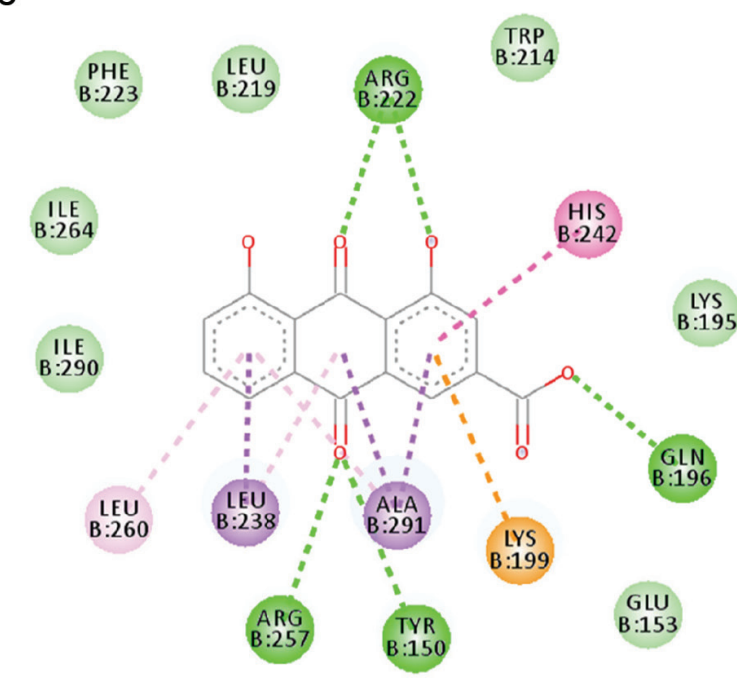

Interactions

van der Waals

Conventional hydrogen bond

Pi-Cation
Pi-Sigma

Pi-Pi T-shaped

Pi-Alkyl

Figure 5. Computational docking of HSA and rhein. The binding site of rhein on HSA (a). The binding mode of rhein and HSA in a zoomed view (b), and illustrations of types of interactions between rhein and HSA (c).

cine 238 and alanine 291. Data from the computational modeling suggest that rhein interacts with HSA by forming hydrogen bonds and increasing the hydrophobicity. These interactions may result in the stabilization of rhein-HSA complex and, thereby, protect HSA against glycation.

Notably, rhein, which is the most well studied among these anthraquinones (Agarwal et al., 2000; Yen et al., 2000), was selected as a 'representative anthraquinone' for further mechanistic studies which is a limitation of the current study. It is possible that structural variations in the other anthraquinones may lead to alterations in their interactions with HSA via different mechanisms. For instance, the antioxidant effects of anthraquinone derivatives have been reported to be impacted by the structural characteristics of their substituents from computational studies (Marković et al., 2016). Therefore, further mechanistic studies to evaluate the interactions of the other six anthraquinone derivatives with HSA is warranted.

In summary, several natural anthraquinone derivatives including aloin, aloe-emodin, chrysophanol, emodin, physcion, and rhein showed inhibitory effects against D-fructose-, MGO-, and GO-induced HSA glycation. Mechanistic studies suggest that anthraquinones may inhibit HSA glycation by maintaining the structures of HSA protein. Furthermore, rhein, used as a representative anthraquinone, was investigated for its interaction with HSA protein. Biophysical assays revealed that rhein binds to HSA protein in a 2:1 ratio and directly interacts with the amino acid residues of the HSA protein. These observations were further supported by the computational docking experiments. Our findings support data that anthraquinones are promising AGEs inhibitors and provide insights for understanding the mechanisms of AGEs inhibition by these natural compounds.

\section{Acknowledgments}

Data were acquired from instruments located at the University of Rhode Island in the RI-INBRE core facility obtained from Grant \# P20GM103430 from the National Center for Research Resources (NCRR), a component of the National Institutes of Health (NIH). R.C. and C.C. was supported by the RI-INBRE Summer Undergraduate Research Fellowship (SURF) program. J.S. was supported by the Yang Fan Innovative \& Entrepreneurial Research Team Project (201312H05) and the 111 Project (B17018).

\section{Conflict of interest}

The authors declare no conflict of interest.

\section{References}

Adisakwattana, S., Jiphimai, P., Prutanopajai, P., Chanathong, B., Sapwarobol, S., and Ariyapitipan, T. (2010). Evaluation of alpha-glucosidase, alpha-amylase and protein glycation inhibitory activities of edible plants. Int. J. Food Sci. Nutr. 61: 295-305.

Agarwal, S.K., Singh, S.S., Verma, S., and Kumar, S. (2000). Antifungal activity of anthraquinone derivatives from Rheum emodi. J. Ethnopharmacol. 72: 43-46.

Anguizola, J., Matsuda, R., Barnaby, O.S., Hoy, K.S., Wa, C., DeBolt, E., Koke, M., and Hage, D.S. (2013). Review: Glycation of human serum albumin. Clin. Chim. Acta 425: 64-76.

Anraku, M., Chuang, V.T.G., Maruyama, T., and Otagiri, M. (2013). Redox 
properties of serum albumin. Biochim. Biophys. Acta-Gen. Subjects 1830: 5465-5472.

Arvindekar, A., More, T., Payghan, P.V., Laddha, K., Ghoshal, N., and Arvindekar, A. (2015). Evaluation of anti-diabetic and alpha glucosidase inhibitory action of anthraquinones from Rheum emodi. Food Funct. 6: 2693-2700.

Bi, S., Song, D., Kan, Y., Xu, D., Tian, Y., Zhou, X., and Zhang, H. (2005). Spectroscopic characterization of effective components anthraquinones in Chinese medicinal herbs binding with serum albumins. Spectrochim. Acta Part A Mol. Biomol. Spectrosc. 62: 203-212.

Bou-Abdallah, F., Sprague, S.E., Smith, B.M., and Giffune, T.R. (2016). Binding thermodynamics of Diclofenac and Naproxen with human and bovine serum albumins: A calorimetric and spectroscopic study. J. Chem. Thermodyn. 103: 299-309.

Choi, S., and Chung, M.H. (2003). A review on the relationship between aloe vera components and their biologic effects. Semin. Integr. Med. 1: 53-62.

Dave, H., and Ledwani, L. (2012). A review on anthraquinones isolated from Cassia species and their applications. Indian J. Nat. Prod. Resour. 3: 291-319.

Ding, F., Zhang, L., Diao, J.X., Li, X.N., Ma, L., and Sun, Y. (2012). Human serum albumin stability and toxicity of anthraquinone dye alizarin complexone: An albumin-dye model. Ecotoxicol. Environ. Safety 79: 238-246.

Eshun, K., and He, Q. (2004). Aloe Vera: A valuable ingredient for the food, pharmaceutical and cosmetic industries - A review. Crit. Rev. Food Sci. Nutr. 44: 91-96.

Fanali, G., Di Masi, A., Trezza, V., Marino, M., Fasano, M., and Ascenzi, P. (2012). Human serum albumin: From bench to bedside. Mol. Aspects Med. 33: 209-290.

He, D., Chen, B., Tian, Q., and Yao, S. (2009). Simultaneous determination of five anthraquinones in medicinal plants and pharmaceutical preparations by HPLC with fluorescence detection. J. Pharm. Biomed. Anal. 49: 1123-1127.

He, X.M., and Carter, D.C. (1992). Atomic structure and chemistry of human serum albumin. Nature 358: 209-215.

Joglekar, M.M., Panaskar, S.N., Chougale, A.D., Kulkarni, M.J., and Arvindekar, A.U. (2013). A novel mechanism for antiglycative action of limonene through stabilization of protein conformation. Mol. BioSyst. 9: 2463-2472.

Kang, S., Zhao, X., Yue, L., and Liu, L. (2017). Main anthraquinone components in Aloe vera and their inhibitory effects on the formation of advanced glycation end-products. J. Food Process. Preserv. 41: e13160.

Koyama, J., Morita, I., and Kobayashi, N. (2007). Simultaneous determination of anthraquinones in rhubarb by high-performance liquid chromatography and capillary electrophoresis. J. Chromatogr. A 1145: 183-189.

Li, Y., Yao, X., Jin, J., Chen, X., and Hu, Z. (2007). Interaction of rhein with human serum albumin investigation by optical spectroscopic technique and modeling studies. Biochim. Biophys. Acta-Proteins Proteomics 1774: 51-58.

Liu, W., Ma, H., DaSilva, N.A., Rose, K.N., Johnson, S.L., Zhang, L., Wan, C., Dain, J.A., and Seeram, N.P. (2016). Development of a neuroprotective potential algorithm for medicinal plants. Neurochem. Int. 100: 164-177.

Liu, W., Ma, H., Frost, L., Yuan, T., Dain, J.A., and Seeram, N.P. (2014). Pomegranate phenolics inhibit formation of advanced glycation endproducts by scavenging reactive carbonyl species. Food Funct. 5: 2996-3004.

Liu, W., Wei, Z., Ma, H., Cai, A., Liu, Y., Sun, J., DaSilva, N.A., Johnson, S.L., Kirschenbaum, L.J., Cho, B.P., Dain, J.A., Rowley, D.C., Shaikh, Z.A., and Seeram, N.P. (2017). Anti-glycation and anti-oxidative effects of a phenolic-enriched maple syrup extract and its protective effects on normal human colon cells. Food Funct. 8: 757-766.
Ma, H., Johnson, S.L., Liu, W., Dasilva, N.A., Meschwitz, S., Dain, J.A., and Seeram, N.P. (2018). Evaluation of polyphenol anthocyanin-enriched extracts of blackberry, black raspberry, blueberry, cranberry, red raspberry, and strawberry for free radical scavenging, reactive carbonyl species trapping, anti-glycation, anti- $\beta$-amyloid aggregation, and microglial neuroprotective effects. Int. J. Mol. Sci. 19: 461.

Ma, H., Liu, W., Frost, L., Kirschenbaum, L.J., Dain, J.A., and Seeram, N.P. (2016). Glucitol-core containing gallotannins inhibit the formation of advanced glycation end-products mediated by their antioxidant potential. Food Funct. 7: 2213-2222.

Ma, H., Liu, W., Frost, L., Wang, L., Kong, L., Dain, J.A., and Seeram, N.P. (2015). The hydrolyzable gallotannin, penta-O-galloyl-beta-D-glucopyranoside, inhibits the formation of advanced glycation endproducts by protecting protein structure. Mol. Biosyst. 11: 1338-1347.

Ma, H., Wang, L., Niesen, D.B., Cai, A., Cho, B.P., Tan, W., Gu, Q., Xu, J., and Seeram, N.P. (2015). Structure activity related, mechanistic, and modeling studies of gallotannins containing a glucitol-core and a-glucosidase. RSC Adv. 5: 107904-107915.

Marković, Z., Jeremić, S., Marković, J.D., Pirković, M.S., and Amić, D. (2016). Influence of structural characteristics of substituents on the antioxidant activity of some anthraquinone derivatives. Comput. Theor. Chem. 1077: 25-31.

Peng, X., Ma, J., Chen, F., and Wang, M. (2011). Naturally occurring inhibitors against the formation of advanced glycation end-products. Food Funct. 2: 289-301.

Raghav, A., and Ahmad, J. (2014). Glycated serum albumin: A potential disease marker and an intermediate index of diabetes control. Diabetes Metab. Syndr.: Clin. Res. Rev. 8: 245-251.

Roche, M., Rondeau, P., Singh, N.R., Tarnus, E., and Bourdon, E. (2008). The antioxidant properties of serum albumin. FEBS Lett. 582: 1783-1787.

Sob, S.V.T., Wabo, H.K., Tchinda, A.T., Tane, P., Ngadjui, B.T., and Ye, Y. (2010). Anthraquinones, sterols, triterpenoids and xanthones from Cassia obtusifolia. Biochem. Syst. Ecol. 38: 342-345.

Sun, J., Liu, W., Ma, H., Marais, J.P.J., Khoo, C., Dain, J.A., Rowley, D.C., and Seeram, N.P. (2016). Effect of cranberry (Vaccinium macrocarpon) oligosaccharides on the formation of advanced glycation endproducts. J. Berry Res. 6: 149-158.

Tang, D., Zhu, J.X., Wu, A.G., Xu, Y.H., Duan, T.T., Zheng, Z.G., Wang, R.S., Li, D., and Zhu, Q. (2013). Pre-column incubation followed by fast liquid chromatography analysis for rapid screening of natural methylglyoxal scavengers directly from herbal medicines: Case study of Polygonum cuspidatum. J. Chromatogr. A 1286: 102-110.

Vargas, F., Rivas, C., and Medrano, M. (2004). Interaction of emodin, aloeemodin, and rhein with human serum albumin: A fluorescence spectroscopic study. Toxicol. Mech. Meth. 14: 227-231.

Wang, N., Su, M., Liang, S., and Sun, H. (2016). Investigation of six bioactive anthraquinones in slimming tea by accelerated solvent extraction and high performance capillary electrophoresis with diode-array detection. Food Chem. 199: 1-7.

Wu, C.H., Huang, S.M., Lin, J.A., and Yen, G.C. (2011). Inhibition of advanced glycation endproduct formation by foodstuffs. Food Funct. 2: 224-234.

Xie, Y., and Chen, X. (2013). Structures required of polyphenols for inhibiting advanced glycation end products formation. Curr. Drug Metab. 14: 414-431.

Yaylayan, V.A., Huyghues-Despointes, A., and Polydorides, A. (1992). A fluorescamine-based assay for the degree of glycation in bovine serum albumin. Food Res. Int. 25: 269-275.

Yen, G.C., Duh, P.D, and Chuang, D.Y. (2000). Antioxidant activity of anthraquinones and anthrone. Food Chem. 70: 437-441.

Yoo, N.H., Jang, D.S., Lee, Y.M., Jeong, I.H., Cho, J.H., Kim, J.H., and Kim, J.S. (2010). Anthraquinones from the Roots of Knoxia valerianoides inhibit the formation of advanced glycation end products and rat lens aldose reductase in vitro. Arch. Pharmacal. Res.33: 209-214. 